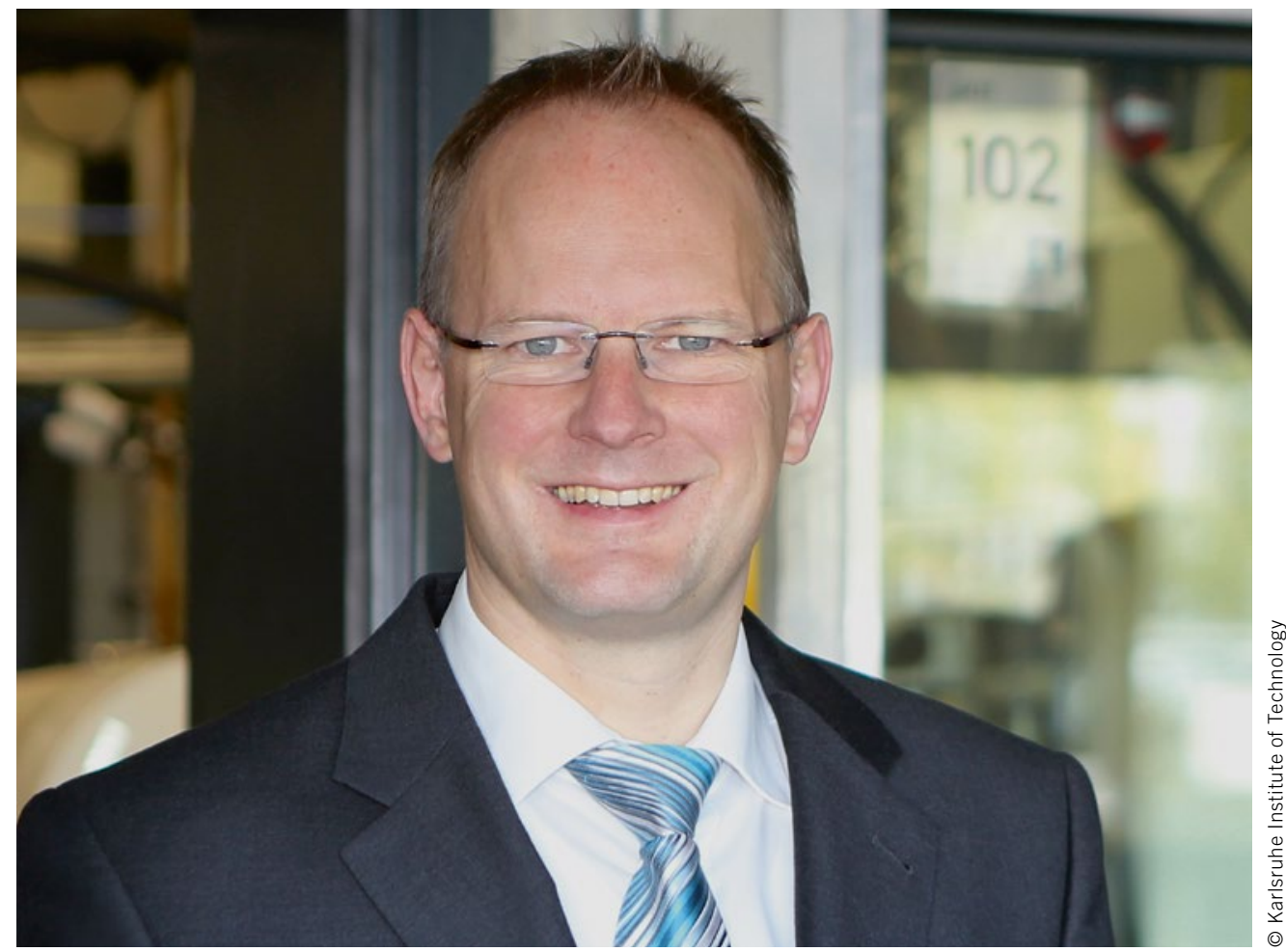

Prof. Dr. sc. techn. Thomas Koch Head of the Institute of Internal Combustion Engines at the Karlsruhe Institute of Technology (KIT)

\title{
A Paradigm Shift in the Automotive Industry
}

Ever since the automobile and combustion engine came into existence, engineers have constantly striven to boost the efficiency of combustion-engine powered drive systems. Recent years have seen great leaps forward and numerous innovations in a delicate balancing act, aiming to boost practical value while also reducing the energy required and level of emissions generated. Moreover, while previous development goals tended to be narrow and centre on meeting regulatory requirements and their consumption and exhaust gas limits, today's engineers have to adopt a whole different perspective. Combustion engine design and its in-vehicle applications are no longer dictated by fixed cycles and clearly defined engine map ranges. Instead, the ultimate task before us is to take all operating conditions into consideration while also introducing legally imposed emission measurement during real operation. Given how crucial measurable variables and clear guidelines are to us, we have to take on the multi-dimensional task of viewing the product while in use and optimising energy consumption and exhaust emissions throughout all feasible usage scenarios. New tools are are being deployed for this purpose for testing the drive system and transferring the application as well as incorporating them into vehicles.
The wide range of tests on a highly dynamic engine test bed through to completion of a full powertrain test, are conducted in combination with total vehicle simulations taking account of surrounding conditions. A complete driving cycle is performed in detail in literally a matter of microseconds, allowing critical dynamic situations to be detected and mastered. These tools are based on an intimate understanding of internal engine processes, which are measured on the spot and their effects examined. This is taking place alongside ongoing development of PEMS measurement technology, which is set to be refined even further to a point where it can provide even more accurate measurement results. Likewise, this measurement technology must be aligned with research measurements tools as well as optical methods used to detect individual specimens with temporal resolution. Measurement fault tolerance, which in the case of the Euro $6 \mathrm{c}$ legislation includes a safety factor of $50 \%$, remains a challenge for developers. Whatever happens, the engines and vehicles developed are sure to keep impressing customers while also playing a key role in boosting the image of the combustion engine with consumption and emission rates that reflect realities. 